\title{
ДИНАМИЧЕСКАЯ КОНФИГУРАЦИЯ ПАРАМЕТРОВ СЕТЕВЫХ СОЕДИНЕНИЙ
}

Корза Егор Александрович

курсант

Краснодарское Высшее Военное Училище

Аннотация: В статье рассматривается вопрос использования протокола динамической конфигурации параметров сетевых соединений. Назначение IPадресов. Взаимодействие сервера и клиента. Безопасность протокола DHCP.

Ключевые слова: DHCP, IP, MAC-адрес, Интернет.

\section{DYNAMIC CONFIGURATION OF NETWORK CONNECTION PARAMETERS}

\section{Korza Egor Aleksandrocvich}

\begin{abstract}
The article discusses the use of the protocol for dynamic configuration of network connection parameters. Assigning IP addresses. Server and client interaction. The security of the DHCP protocol.
\end{abstract}

Key words: DHCP, IP, MAC address, Internet.

Интернет представляет собой совокупность соединенных между собой компьютерных сетей, в которых используются единые согласованные правила обмена данными между компьютерами. Интернет объединяет тысячи глобальных региональных и локальных сетей, рассеянных по всему земному шару. Наиболее популярным сервисом, представленным в Интернете, является WWW («World Wide Web, буквально — всемирная паутина), но говоря «WWW» и «Интернет», мы имеем в виду не одно и тоже. WWW - это информационный ресурс, некий мир знаний, распределенный по компьютерам во всей сети, в то время как сам Интернет является средством передачи информации между компьютерами, подобно тому, как телефонная сеть есть средство передачи информации между говорящими. Кроме WWW в Интернете предоставляются и другие услуги. 


\section{МОЛОДЕЖНАЯ НАУКА КАК ФАКТОР И РЕСУРС \\ ОПЕРЕЖАЮЩЕГО РАЗВИТИЯ}

Сам по себе Интернет не имеет никакого собственника, хотя, конечно, каждая входящая в него сеть принадлежит какой-либо компании, некоммерческой или государственной организации. Не существует также и специального органа управления, который бы контролировал всю работу Интернета.

На заре своего развития компьютерные сети существовали независимо друг от друга, решая конкретные задачи для конкретных групп пользователей. В соответствии с этими задачами выбирались те или иные сетевые технологии, программы и оборудование. Построить универсальную компьютерную сеть мирового масштаба из однотипной аппаратуры просто невозможно, поскольку такая сеть не могла бы удовлетворять потребности всех её потенциальных пользователей. Одним нужна высокоскоростная сеть для соединения машин в пределах здания, а другими - надежные коммуникации между компьютерами, разнесенными на сотни километров; одни используют мощные мэйнфреймы, другие - персональные компьютеры.

Тогда возникла идея объединить множество физических сетей и разнородных компьютеров в единую глобальную сеть с помощью набора специальных стандартных «правил», их называют протоколами, которые не зависели бы от типа оборудования и программного обеспечения.

Протокол DHCP - протокол динамической конфигурации хоста, предназначен для автоматической настройки параметров стека TCP/IP хоста в момент его загрузки или по требованию пользователя. Параметры стека выдаются хосту сервером DHCP по запросу поступившему от хоста. При этом хост получает IP-адрес и сетевую маску, а также адрес маршрутизатора по умолчанию, адрес сервера DNS, имя своего домена DNS и другие параметры в зависимости от конфигурации сервера.

Протокол DHCP позволяет существенно упростить и упорядочить использование IP-адресов и конфигурирование компьютеров в корпоративных сетях, особенно - в крупных.

Назначение IP-адреса хосту может быть динамическим и статическим. В первом случае сервер выдает хосту любой свободный адрес из блока (пула) специально зарезервированных адресов. При статическом назначении хост получает адрес, заранее назначенный ему администратором сети (для этого хост должен предъявить DHCP-серверу какой-нибудь уникальный идентификатор, например свой МАC-адрес). 


\section{МОЛОДЕЖНАЯ НАУКА КАК ФАКТОР И РЕСУРС ОПЕРЕЖАЮЩЕГО РАЗВИТИЯ}

Хост поможет использовать полученные параметры в течение указанного сервером времени (этот промежуток называется сроком аренды-lease time). По истечении времени аренды хост должен получить новые параметры конфигурации (либо сервер подтвердит уже имеющееся назначение)

Протокол DHCP использует транспортный протокол UDP для передачи сообщений между клиентом и сервером (порт сервера - 67, клиента - 68).

Взаимодействие сервера и клиента происходит в следующем порядке.

Клиент, используя широковещательный IP-адрес получателя и нулевой адрес отправителя, рассылает в своей сети сообщение DHCPDISCOVER, в которое он может включить желаемые параметры конфигурации (IP-адрес, срок аренды и т.п.)

Сервер получает запрос и откликается предложением (DHCPOFFER), содержащим предлагаемый хосту IP-адрес и другие конфигурационные данные; адрес, содержащийся в предложении, временно положительного или отрицательного ответа от хоста или до истечения тайм-аута. Предварительно сервер должен проверить, не используется ли предлагаемый адрес каким-то другим хостом-например, с помощью сообщений ICMP «Echo» (команда ping).

Отправляя сообщение DHCPOFFER, сервер устанавливает IP-адрес получателя в соответствии со сделанным предложением, a MAC-адрес получателя устанавливается равным МАC-адресу клиента (он извлекается из кадра, в котором клиент прислал сообщение DHCPDISCOVER). Таким образом, сообщение будет доставлено клиенту на уровень доступа к сети, извлечено из кадра и передано модулю IP. Предполагается, что IP-модуль клиента на этой стадии способен принять дейтаграмму, направленную на его МАC-адрес, несмотря на то, что IP-адрес еще не сконфигурирован. Если такой возможности нет, то клиент в своих сообщениях должен устанавливать специальный бит BROADCAST, на основании чего сервер будет отвечать широковещательно.

Клиент получает предложение DHCPOFFER. Возможно, что в сети работает несколько DHCP-серверов, тогда клиент может получить несколько предложений. Если ни одного предложения в течение определенного времени не получено, то клиент начинает процесс заново. Получив предложение, клиент на основании своих настроек решает принять предложение определенного сервера (или принять первое поступившее предложение, если никаких настроек нет). Клиент широковещательно рассылает сообщение DHCPREQUEST, в котором указывает идентификатор (адрес) сервера, предложение которого он 
принял, предложенный сервером IP-адрес и другие конфигурационные параметры.

Сообщение DHCPREQUEST получают все DHCP-серверы. Те из них, кт не обнаружит своего идентификатора в этом сообщении, воспримут его как отказ клиента от сделанного предложения. Выбранный же клиентом сервер закрепляет за клиентом требуемый адрес, устанавливает срок аренды и отвечает сообщением DHCPACK, содержащим окончательные параметры и адрес клиента. Это сообщение отправляется тем же способом, как и DHCPOFFER.

Если же сервер по какой-то причине не может удовлетворить запрос клиента (например, DHCPRQUEST задержался, и сервер уже выдал адрес другому клиенту), то сервер отвечает отказом DHCONACK [1. C. 103].

Клиент получает сообщение DHCPACK и проверяет, не выделен ли уже полученный адрес другому хосту (проверка производится посылкой ARPзапроса). Если проверка прошла успешно, то клиент конфигурирует себя в соответствии с полученными параметрами. Если полученный адрес уже используется, то клиент отправляет серверу сообщение DHCPDECLINE. В этом случае, а также при получении отказа DHCPNACK или при отсутствии реакции сервера после нескольких повторных посылок DHCPREQUEST клиент начинает весь процесс заново.

После завершения работы клиент может освободить занимаемый адрес путем отправления серверу сообщения DHCPRELEASE.

Если перед началом процесса получения адрес клиент помнит свой предыдущий адрес и конфигурационные параметры, полученные у DHCPсервера, и хочет использовать их вновь, то он может сократить процесс конфигурации, начав сразу с посылки сообщения DHCPREQUEST. Если при этом клиент не получает никакого отклика от сервера (серверов) и срок аренды предыдущей конфигурации еще не истек, клиент может использовать эту конфигурацию, предварительно проверив, что адрес свободен.

Один DHCP-сервер может работать в нескольких сетях. Для этого в каждой сети должен быть сконфигурирован DHCP-relay (или BOOTP-relay) специальный посредник, который будет ретранслировать сообщения между сервером и клиентом. Без посредника DHCP-сервер не получит запросов, так как широковещательные IP-дейтаграммы не выходят за пределы IP-сети.

Что касается безопасности протокола DHCP - никаких мер безопасности в протоколе DHCP не предусмотрено. Это значит, что злоумышленник может 


\section{МОЛОДЕЖНАЯ НАУКА КАК ФАКТОР И РЕСУРС \\ ОПЕРЕЖАЮЩЕГО РАЗВИТИЯ}

установить в сети ложный DHCP-сервер и конфигурировать хосты так, что они будут пользоваться ложным маршрутизатором, ложным DNS-сервером или вообще утратят способность к соединению.

Некоторые DHCP-клиенты позволяют указать в своей конфигурации IPадреса DHCP-серверов, от которых они будут принимать предложения, а предложения остальных серверов игнорировать. Однако, во-первых, необходимость локального конфигурирования каждого клиента идет вразрез с самым смыслом использования DHCP; во-вторых, установка злоумышленником в своих DHCP-сообщения не является проблемой для злоумышленника.

Оборотная сторона отсутствия мер безопасности в DHСР-ложные клиенты. Злоумышленник, не санкционировано подключившись к сети с сервером DHCP, легко может получить все необходимые настройки TCP/IP для этой сети и легальный IP-адрес для своего компьютера. Более того, в некоторых случаях путем многократной посылки запросов якобы от разных хостовклиентов злоумышленник может оттянуть на себя адресные ресурсы пула, тем самым заблокировав конфигурирование легитимных клиентов.

В случае когда МАC-адреса всех компьютеров сети с DHCP-сервисом известны и не меняются слишком часто (что в большинстве случаев верно для локальных сетей организаций) администратору производить назначение IPадресов и выдачу конфигурационной информации только зарегистрированным хостам. Отметим, что сервер получает MAC-адрес в специальном поле DHCPзапроса, а не извлекает из кадра Ethernet, в котором запрос прибыл от клиента (иначе нельзя было бы использовать ретрансляторы). Это позволяет злоумышленник, желающему нелегально сконфигурировать свой хост, заявить в своем запросе MAC-адрес зарегистрированного клиента. Для отслеживания таких ситуаций необходимо применять статическую ARP-таблицу и отслеживать нарушения путем прослушивания сети или соответствующей настройки коммутаторов.

Мир Интернета, или как его еще называют «виртуальный мир», не слишком сильно отличается от мира «реального». Мы все привыкли к тому, что, выходя из дома, мы закрываем дверь на замок, не оставляем ценности в гардеробе, устанавливаем на автомобили противоугонные устройства - это рутинные и, к сожалению необходимые меры безопасности. Несмотря на недостатки и частые доработки, протокол DHCP широко используется в современных сетях. 


\section{Список литературы}

1. Мамаев М., Петренко С.: Технологии защиты информации в интернете. Специальный справочник. - Спб.: Питер, 2002.-848 с. 